\title{
Correlation energy in a triplet state of a two-electron spherical quantum dot
}

\author{
A. Rejo Jeice* and K. Navaneethakrishanan ${ }^{\dagger}$ \\ School of Physics, Madurai Kamaraj University,Madurai-625021,India.
}

(Received on 16 November, 2008)

\begin{abstract}
Correlation energies in the (1s-1p) triplet state of a two-electron spherical QD with square-well potential confinement are estimated for dots of different radii. The results are presented taking GaAs dot as an example. Our results show that the correlation energies are i) negative in a triplet state in contrast to the singlet state, ii)approaches zero as the dot size approaches infinity, and iii) the "fictitious crossing" of the singlet and triplet state energies at a particular dot size is explained on the basis of Hund's rules.
\end{abstract}

Keywords: Spherical quantum dot; Square well confinement; Correlation energies; Singlet and Triplet states

\section{INTRODUCTION}

Energy levels of the carrier in a QD exhibit discrete spectra as in an atom. Hence QD is called an artificial atom [1,2].It has been shown that electron correlation plays a significant role in QD's. Such correlations lead to interesting phenomena such as Coulomb blockade [3], which is exploited in single electron transistors [4]. There have been considerable efforts on the estimation of correlations in few electron QD's [5,6]. Though most of these works are on QD with parabolic confinement [7], some authors have investigated the correlation effects under square well confinement also [8]. Recently Sivakami et al. [9] have considered electron correlation in a singlet ground state of a two electron spherical QD with finite barrier square well confinement. Unlike certain works which involve extensive numerical work, these authors obtain closed analytical expressions by treating electron correlations by a perturbation method, which utilizes the evaluation of certain integrals by the mean value theorem [10].

The purpose of the present work is two-fold. The first is that we are treating a triplet state $(1 \mathrm{~s}-1 \mathrm{p})$ by extending the calculations of reference [9]. Secondly we point out that the crossing of the singlet and triplet state energies at a particular dot radius as observed in Szafran et al. [7] is not fictitious but has a physical basis.

The plan of the presentation of the present work is as follows. In Section 2 we present the mathematical models and the details of calculations. The results obtained are presented in Section 3 with a detailed discussion of the results. Conclusions are presented in Section 4.

\section{MODELS AND CALCULATIONS}

\subsection{Single electron in a spherical quantum dot}

Here we consider a single electron in a Spherical QD in the finite barrier model. In the absence of impurity, within the effective mass approximation, the Hamiltonian is given

*Electronic address: rejojeice@gmail.com

${ }^{\dagger}$ Electronic address: drknk2003@yahoo.co.in by

$$
H_{1}=-\frac{\hbar^{2}}{2 m^{*}} \nabla^{2}+V_{D}(r)
$$

where $m^{*}$ is the effective mass of the electron at the conduction band minimum, which is $0.067 m_{0}$ for GaAs [11], where $m_{0}$ is the free electron mass. In our numerical calculations we use atomic units in which $m_{0}=e^{2}=\hbar^{2}=1$. The confining potential $V_{D}(r)$ is given by,

$$
V_{D}(r)=\mid \begin{array}{ll}
0 & r<R \\
V_{0}=Q_{c} \Delta E_{g}(x) & r>R
\end{array}
$$

where $V_{0}$ is the barrier height, $Q_{c}$ is the conduction band offset parameter which is taken as 0.658 [12]. The band gap difference depends of the concentration of Al. In our case $G a_{1-x} A l_{x} A_{s}$ is the barrier medium in which GaAs dot is embedded. The total energy difference [13] between the well and barrier media, as a function of $x$, is given by

$$
\Delta E_{g}(x)=1.155 x+0.37 x^{2} e V
$$

In the present work we have chosen $x=0.2$, and the value of $V_{0}$ turns to be $161.73 \mathrm{meV}$. Two lowest lying bound states are given by

$$
\begin{aligned}
& \psi_{1 s}(\vec{r})=\mid \begin{array}{ll}
N_{1} \frac{\sin \left(\alpha_{1} r\right)}{\alpha_{1} r} & r \leq R \\
A_{1} \frac{e^{-\beta_{1} r}}{\beta_{1} r} & r \geq R \text { and }
\end{array} \\
& \psi_{1 p}(\vec{r})=\mid \begin{array}{ll}
N_{2}\left[\frac{\sin \left(\alpha_{2} r\right)}{\left(\alpha_{2} r\right)^{2}}-\frac{\cos \left(\alpha_{2} r\right)}{\left(\alpha_{2} r\right)}\right] \cos \theta & r \leq R \\
i A_{2}\left[\frac{1}{\beta_{2} r}+\frac{1}{\left(\beta_{2} r\right)^{2}}\right] e^{-\beta_{2} r} \cos \theta & r \geq R
\end{array}
\end{aligned}
$$

where $N_{1}$ and $N_{2}$ are normalization constants and $\alpha_{1}$ and $\beta_{1}$ are given by $\alpha_{1}=\sqrt{2 m^{*} E}$ and $\beta_{1}=\sqrt{2 m^{*}\left(V_{0}-E\right)}$. Matching the wave function and their derivatives at the boundary $r=R$,we get

$$
A_{1}=N_{1} \sin \left(\alpha_{1} R\right) e^{\beta_{1} R}
$$

$$
A_{2}=-i N_{2}\left(\frac{\beta_{2}}{\alpha_{2}}\right)^{2}\left(\frac{\sin \left(\alpha_{2} R\right)-\alpha_{2} R \cos \left(\alpha_{2} R\right)}{\beta_{2} R+1}\right) e^{\beta_{2} R}
$$


The energy eigenvalues are determined by imposing the Ben Daniel and Duke boundary condition [14]

$$
-\left.\frac{i \hbar}{m_{1}^{*}} \frac{\partial \psi}{\partial r}(r \leq R)\right|_{r=R}=-\left.\frac{i \hbar}{m_{2}^{*}} \frac{\partial \psi}{\partial r}(r \geq R)\right|_{r=R}
$$

We obtain

$$
\alpha_{1} R+\beta_{1} R \tan \left(\alpha_{1} R\right)=0 \quad \text { for } s \text {-states and }
$$

$$
\frac{\cot \left(\alpha_{2} R\right)}{\alpha_{2} R}-\frac{1}{\left(\alpha_{2} R\right)^{2}}=\frac{1}{\beta_{2} R}+\frac{1}{\left(\beta_{2} R\right)^{2}} \quad \text { for } p \text {-states }
$$

If $m_{1}^{*}=m_{2}^{*}=\mathrm{m}^{*}$, solving these transcendental equations numerically, the confined energies $E_{l}^{n}(\mathrm{n}=1,2,3 \ldots ; \ell=0,1)$ are obtained. For other excited states similar equations may be obtained when $\ell=2,3, \ldots$ The confined energy for the first two states are given in Table 1.

TABLE 1: Confined electron energies $(\mathrm{meV})$ in the spherical QD

\begin{tabular}{|c|c|c|c|c|c|}
\hline \multirow{2}{*}{$\begin{array}{c}\text { Dot } \\
\text { Radius }(n m)\end{array}$} & \multicolumn{3}{|c|}{ Confined Energy $(\mathrm{meV})$} & $\begin{array}{c}\text { Correlation } \\
\text { Energy } \\
(\mathrm{meV})\end{array}$ & $\begin{array}{c}\text { Total } \\
\text { Confined } \\
\text { energy (meV) }\end{array}$ \\
\cline { 2 - 4 } & $\left(E_{l s}\right)$ & $\left(E_{l p}\right)$ & $\mathrm{E}_{l s}+\mathrm{E}_{l p}$ & & \\
\hline 10 & 39.33 & 81.49 & 120.82 & -9.97 & 110.85 \\
\hline 15 & 19.66 & 40.80 & 60.46 & -6.31 & 54.15 \\
\hline 20 & 11.73 & 24.28 & 36.01 & -4.62 & 31.39 \\
\hline 25 & 7.79 & 15.90 & 23.69 & -3.63 & 20.06 \\
\hline 30 & 5.53 & 11.31 & 16.84 & -3.00 & 13.84 \\
\hline
\end{tabular}

\subsection{Two electrons in a spherical quantum dot}

The Hamiltonian of the system with interaction is given by

$$
H=\frac{p_{1}^{2}}{2 m^{*}}+\frac{p_{2}^{2}}{2 m^{*}}+V_{D}\left(\overrightarrow{r_{1}}\right)+V_{D}\left(\overrightarrow{r_{2}}\right)+\frac{e^{2}}{\varepsilon_{0}\left|\overrightarrow{r_{1}}-\overrightarrow{r_{2}}\right|}
$$

$$
H=H_{1}+H_{2}+H^{1}, \quad \text { with } \quad H^{1}=e^{2} / \varepsilon_{0}\left|\vec{r}_{1}-\vec{r}_{2}\right|
$$

In the two-electron system we calculate the correlation energy using the perturbation method. Here $H^{1}$ is the perturbation term and $\varepsilon_{0}$ is the static dielectric constant. In our problem we have considered one electron in the ground state (i.e. $1 \mathrm{~s}$-state) and another in the excited state (1p-state). Therefore we may obtain a triplet state and the total spin of the system is 1 . In this situation the wave function of the triplet state is

$$
\psi_{A}\left(\vec{r}_{1}, \vec{r}_{2}\right)=\frac{1}{\sqrt{2}}\left[\psi_{1 s}\left(\vec{r}_{1}\right) \psi_{1 p}\left(\vec{r}_{2}\right)-\psi_{1 s}\left(\vec{r}_{2}\right) \psi_{1 p}\left(\vec{r}_{1}\right)\right]
$$

This is spatially anti-symmetric. In Eq. (11) $\psi_{1 s}\left(r_{i}\right)$ and $\psi_{1 p}\left(r_{i}\right)$ are (with $\left.\mathrm{i}=1,2\right)$ as given in equations (4) and (5). We obtain

$$
\begin{aligned}
& \int \psi_{A}^{*}\left(\vec{r}_{1}, \vec{r}_{2}\right) \psi_{A}\left(\vec{r}_{1}, \vec{r}_{2}\right) d \tau_{1} d \tau_{2} \\
& =\left(\frac{3 R}{2 \alpha_{1}^{2}}-\frac{3 \sin \left(2 \alpha_{1} R\right)}{4 \alpha_{1}^{3}}-\frac{3 \cdot e^{-2 \beta_{1} R}}{2 \beta_{1}^{3}}\right) *\left(\begin{array}{l}
\frac{2}{R^{2}}\left(\frac{R}{2 \alpha_{2}^{4}}-\frac{\sin \left(2 \alpha_{2} R\right)}{4 \alpha_{2}^{5}}\right)-\frac{\sqrt{2}}{R}\left(\frac{1-\cos \left(2 \alpha_{2} R\right)}{2 \alpha_{2}^{4}}\right)+\frac{R}{2 \alpha_{2}^{2}} \\
+\frac{\sin \left(2 \alpha_{2} R\right)}{4 \alpha_{2}^{3}}+\frac{e^{-2 \beta R}}{\beta_{2}^{3}}\left(\frac{1}{2}+\frac{1}{\sqrt{5} \beta_{2} R}+\frac{1}{10 \beta_{2}^{2} R^{2}}\right)
\end{array}\right)
\end{aligned}
$$

\subsection{Correlation Energy}

We calculate the Columbic interaction energy by using perturbation method. The first-order energy is given by

$$
\Delta E^{(1)}=\int \psi_{A}^{*}\left(\vec{r}_{1}, \vec{r}_{2}\right) \frac{e^{2}}{\varepsilon_{0}\left|\vec{r}_{1}-\vec{r}_{2}\right|} \psi_{A}\left(\vec{r}_{1}, \vec{r}_{2}\right) d \vec{r}_{1} d \vec{r}_{2}
$$

were $\psi_{A}\left(\vec{r}_{1}, \vec{r}_{2}\right)$ is given in Eq. (11). After a lengthy and straight forward algebra we obtain,the first-order correction, using

$$
\Delta E^{(1)}=\frac{\left(\psi, H^{1} \psi\right)}{(\psi, \psi)}
$$

which is given in Eq. (15).

$$
\Delta E^{(1)}=\frac{4 \pi e^{2}}{3 \varepsilon_{0}}\left(\frac{N r}{D r}\right)
$$

Where Nr.=Term1+Term2, with Term $1=\sum_{i=1}^{12} C N_{i}$ and Term $2=\sum_{i=13}^{15} C N_{i}$,Here,

$$
\begin{aligned}
& C N_{1}=\left(1-C_{3}\right)\left(\frac{0.0922}{\alpha_{1}^{2} \cdot D S}-\frac{0.1611}{\alpha_{2}^{2} \cdot D S}-\frac{0.0443}{\alpha_{1}^{2} \cdot \alpha_{2}^{2}}\right) \times \\
& \times\left(\frac{1}{\alpha_{1}^{2} \cdot \alpha_{2}^{2} \cdot R^{3}}\right) \\
& C N_{2}=\left(1-C_{4}\right)\left(\frac{0.0649}{A S}-\frac{0.1768}{A D}\right)\left(\frac{1}{\alpha_{1}^{2} \cdot \alpha_{2}^{4} \cdot R^{3} \cdot A D}\right) \\
& C N_{3}=(1-C D)\left(\frac{0.0806}{\alpha_{1}^{2} \cdot \alpha_{2}^{4} \cdot R^{3} \cdot A D^{2}}\right) \\
& C N_{4}=\frac{\left((0.1768)\left(C_{2}^{2}-S_{2}^{2}\right)-0.0962-0.0806 \cdot C S\right)}{\alpha_{1}^{2} \cdot \alpha_{2}^{4} \cdot R^{3} \cdot A S^{2}} \\
& C N_{5}=0.1287 \cdot\left(\left(\frac{1-C S}{A S^{3}}\right)-\left(\frac{1-C D}{A D^{3}}\right)\right) \times
\end{aligned}
$$




$$
\begin{aligned}
& \times\left(\frac{1}{\alpha_{1}^{2} \cdot \alpha_{2}^{3} \cdot R^{3}}\right) \\
& C N_{6}=\frac{0.0101\left(\left(C_{3}+C_{4}\right)-0.4944\right)}{\alpha_{1}^{2} \cdot \alpha_{2}^{2} \cdot R \cdot D S} \\
& C N_{7}=\left(-0.0833 \cdot\left(\frac{S S}{A S^{3}}+\frac{S D}{A D^{3}}\right) \cdot\left(\frac{1}{\alpha_{1}^{2} \cdot \alpha_{2}^{2} \cdot R^{2}}\right)\right) \\
& C N_{8}=\frac{(0.1616+0.0050 \cdot C S)}{\alpha_{1}^{2} \cdot \alpha_{2}^{2} \cdot R \cdot A S^{2}} \\
& C N_{9}=\frac{(0.1616+0.0050 \cdot C D)}{\alpha_{1}^{2} \cdot \alpha_{2}^{2} \cdot R \cdot A D^{2}} \\
& C N_{10}=0.0704 \cdot\left(\frac{S D}{A D^{2}}-\frac{S S}{A S^{2}}\right) \cdot\left(\frac{1}{\alpha_{1}^{2} \cdot \alpha_{2}^{3} \cdot R^{2}}\right)
\end{aligned}
$$

$$
\begin{aligned}
& C N_{11}=2 \cdot S_{1} \cdot C_{1} \cdot\left(\frac{0.0576}{D S}-\frac{0.25 \cdot \alpha_{1}}{\alpha_{2}^{2} \cdot A D}-\frac{0.125}{A S^{2}}-\frac{0.125}{A D^{2}}\right) \times \\
& \times\left(\frac{1}{\alpha_{1}^{3} \cdot \alpha_{2}^{2} \cdot R^{2}}\right) \\
& C N_{12}=2 \cdot S_{2} \cdot C_{2} \cdot\left(\frac{0.4424}{D S}-\frac{0.125}{A D^{2}}-\frac{0.375}{A S^{2}}\right) \times \\
& \times\left(\frac{1}{\alpha_{1}^{2} \cdot \alpha_{2}^{3} \cdot R^{2}}\right)
\end{aligned}
$$

$$
\begin{aligned}
& C N_{13}=\left(\frac{F_{1}}{A S}+\frac{F_{3}}{A D}\right) \cdot\left(\left(\frac{F_{4} \cdot\left(\alpha_{2}^{-1}-A D^{-1}\right)}{R \cdot A D}\right)-\left(\frac{F_{2} \cdot\left(\alpha_{2}^{-1}-A S^{-1}\right)}{R \cdot A S}\right)+\left(\frac{F_{1}}{A S}+\frac{F_{3}}{A D}\right)\right) \cdot\left(\frac{0.3333}{\alpha_{1}^{2} \cdot \alpha_{2}^{2} \cdot R}\right) \\
& C N_{14}=\left(\frac{F_{4}}{A D}-\frac{F_{2}}{A S}\right) \cdot\left(\left(\frac{F_{4}}{\alpha_{2} \cdot R \cdot A D}-\frac{F_{4}}{A D^{2} \cdot R}\right)-\left(\frac{F_{2}}{\alpha_{2} \cdot R \cdot A S}+\frac{F_{2}}{R \cdot A S^{2}}\right)+\left(\frac{F_{1}}{A S}+\frac{F_{3}}{A D}\right)\right) \cdot\left(\frac{0.3849}{\alpha_{1}^{2} \cdot \alpha_{2}^{3} \cdot R^{2}}\right)
\end{aligned}
$$

and

$$
C N_{15}=E_{1} \cdot E_{2} \cdot\left(0.0211+\frac{0.109}{\beta_{2} \cdot R}+\frac{0.1}{R \cdot B S}+\frac{0.045}{\beta_{2} \cdot R^{2} \cdot B S}\right)
$$

Dr. is given by

$$
\text { Dr. }=D_{1}\left[D_{2}-D_{3}+D_{4}+D_{5}+D_{6}\right]
$$

Where

$$
\begin{gathered}
D_{1}=\left(\frac{3 R}{2 \alpha_{1}^{2}}-\frac{3 S_{1}}{4 \alpha_{1}^{3}}-\frac{3 E_{1}}{2 \beta_{1}^{3}}\right), \\
D_{2}=\frac{2}{R^{2}}\left(\frac{R}{2 \alpha_{2}^{4}}-\frac{S_{2}}{4 \alpha_{2}^{5}}\right), \\
D_{3}=\left(\frac{1-C_{2}}{\sqrt{2} \cdot \alpha_{2}^{4} \cdot R}\right) \\
D_{4}=\left(\frac{R}{2 \alpha_{2}^{2}}\right), \quad D_{5}=\left(\frac{S_{2}}{4 \alpha_{2}^{3}}\right) \text { and } \\
D_{6}=\frac{E_{2}}{\beta_{2}^{3}}\left(0.5+\frac{0.45}{\beta_{2} R}+\frac{0.1}{\beta_{2}^{2} R^{2}}\right)
\end{gathered}
$$

In the above expression. $S_{i}=\sin \alpha_{i} R, C_{i}=\cos \alpha_{i} R, E_{i}=$ $\exp \left(2 \beta_{i} R\right)$ with $\mathrm{i}=1$ and 2

$$
\begin{aligned}
C_{3} & =\cos \left(2 \alpha_{1} R\right), C_{4}=\cos \left(2 \alpha_{2} R\right), \\
A D & =\alpha_{1}-\alpha_{2}, A S=\alpha_{1}+\alpha_{2}, B D=\beta_{1}-\beta_{2}, \\
B S & =\beta_{1}+\beta_{2}, D S=\alpha_{1}^{2}-\alpha_{1}^{2}, S D=\sin (2 A D \cdot R), \\
S S & =\sin (2 A S \cdot R), \\
C D & =\cos (2 A D \cdot R), C S=\cos (2 A S \cdot R), \\
F_{1} & =\cos (A S \cdot R), \\
F_{2} & =\sin (A S \cdot R) “ F_{3}=\cos (A D \cdot R), F_{4}=\sin (A D \cdot R) "
\end{aligned}
$$

We have used the rms values $\frac{\sqrt{2}}{R}$ for $\frac{1}{r_{1}}$ and $\frac{1}{r_{2}}$ in certain integrals by using the mean value theorem [10] as in Ref. [9]. The correlation energies are obtained for different dot radii as shown in Table 1.

\section{RESULTS AND DISCUSSION}

From Table 1 we find that as the dot size increases, the confinement energy decreases for both the states. Also we find that the confinement energy of the p-state is approximately twice as that of the s-state, as in Ref[9].We also notice that the confinement energy decreases as the dot size increases, which is well known in the literature [7,9]. We have also shown the correlation energy. The magnitude of the first-order correction decreases as the dot size increases showing that correlation is important in nanosystems.

TABLE 2: Comparison of singlet and triplet state energies.

\begin{tabular}{|c|c|c|c|c|c|c|}
\hline $\begin{array}{c}\text { Dot } \\
\text { Radius } \\
(\mathrm{nm})\end{array}$ & \multicolumn{2}{|c|}{$\begin{array}{c}\text { Confined energy } \\
(\mathrm{meV})\end{array}$} & \multicolumn{2}{c|}{$\begin{array}{c}\text { Correlation energy } \\
(\mathrm{meV})\end{array}$} & \multicolumn{2}{|c|}{$\begin{array}{c}\text { Total confined energy } \\
(\mathrm{meV})\end{array}$} \\
\cline { 2 - 7 } & singlet & triplet & singlet & triplet & singlet $^{\dagger}$ & Triplet \\
\hline 10 & 78.66 & 120.82 & 15.56 & -9.97 & 94.23 & 110.85 \\
\hline 15 & 39.32 & 60.46 & 10.38 & -6.31 & 49.70 & 54.15 \\
\hline 20 & 23.46 & 36.01 & 7.78 & -4.62 & 31.24 & 31.39 \\
\hline 25 & 15.58 & 23.69 & 6.23 & -3.63 & 21.81 & 20.06 \\
\hline 30 & 11.06 & 16.84 & 5.19 & -3.00 & 16.25 & 13.84 \\
\hline
\end{tabular}

${ }^{\dagger}$ From Ref.[9]

The correlation value is negative as expected. This is 
a consequence of the exchange interaction in the triplet state which is Columbic in origin and has no classical analogue.This interaction arises because of the antisymmetric nature of the wave functions Eq. (11). The spin part,though not considered explicitly here, should be symmetric for fermions, and corresponds to a triplet state.There is no exchange interaction among electrons of opposite spin orientations. This interaction is attractive as in ferromagnetism via Heisenberg model which uses spins explicitly. It is a direct confirmation of the Hund's rule which favors parallel arrangement of spins in the various atomic sub-shells consistent with maximum spin value. In contrast, in the singlet state, the correlation energy is repulsive as seen in Fig. 1.

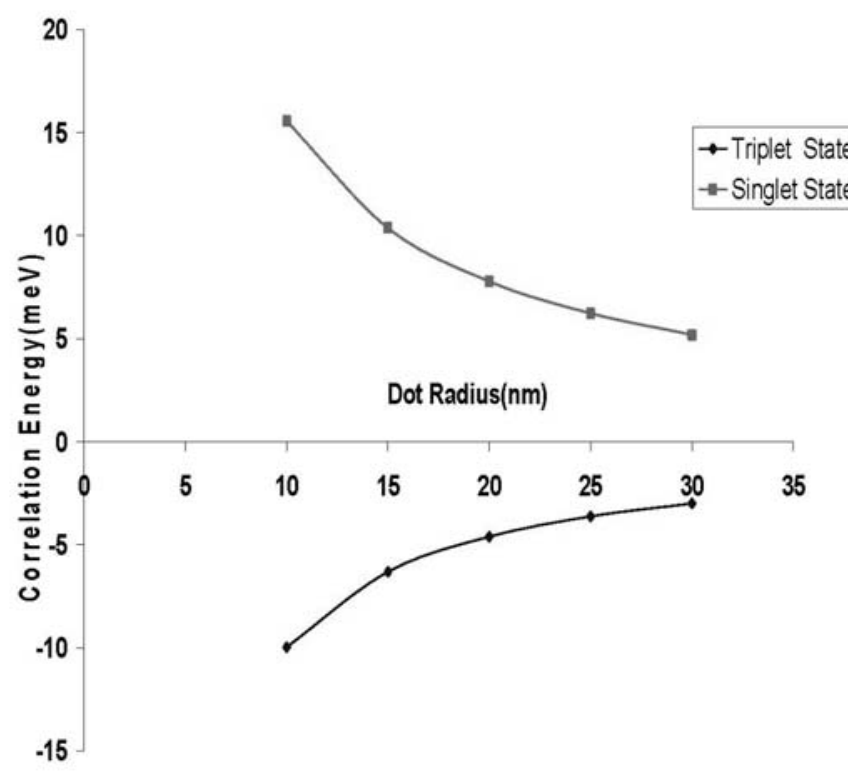

FIG. 1: Correlation Energy Vs Dot Radius for Singlet and Triplet States.

When we compare the magnitudes of the correlation energies of the triplet state with the singlet state, it is seen from Table 2 that the singlet state energies are lower than the corresponding triplet state energies. The singlet state energies are taken from Ref. [9].

The first-order correction itself is negative and is small (about 8\%).So the second-order correction is negligible and the evaluation is also tedious. Even if it is possible for dots of larger radii, the correlation is still smaller, as seen from Table 1.

We notice that the confined energy difference between the singlet and triplet states for narrow dots is about $17 \%$.This is in agreement with the results of Ref. [15] where another quantum well system with parabolic confinement is considered. For larger dots the percentage of confinement also decreases and it seems to be more or less the same for both the states.

The confined energies for the singlet and triplet states for different dot radii are shown in Fig. 2. From this figure it follows that for dot radii less than $20 \mathrm{~nm}$,the singlet state has the lowest energy, in support of the general principle that the energy of the system should be the lowest; for dot radii greater than $20 \mathrm{~nm}$, the triplet state has the lowest energy. This is because as the dot radius increases the energy separation between the $1 \mathrm{~s}$ and $1 \mathrm{p}$-states reduce and $2 E_{1 s} \approx E_{1 s}+E_{1 p}$. But the exchange energy is attractive. Hence the triplet state energy lies lower.

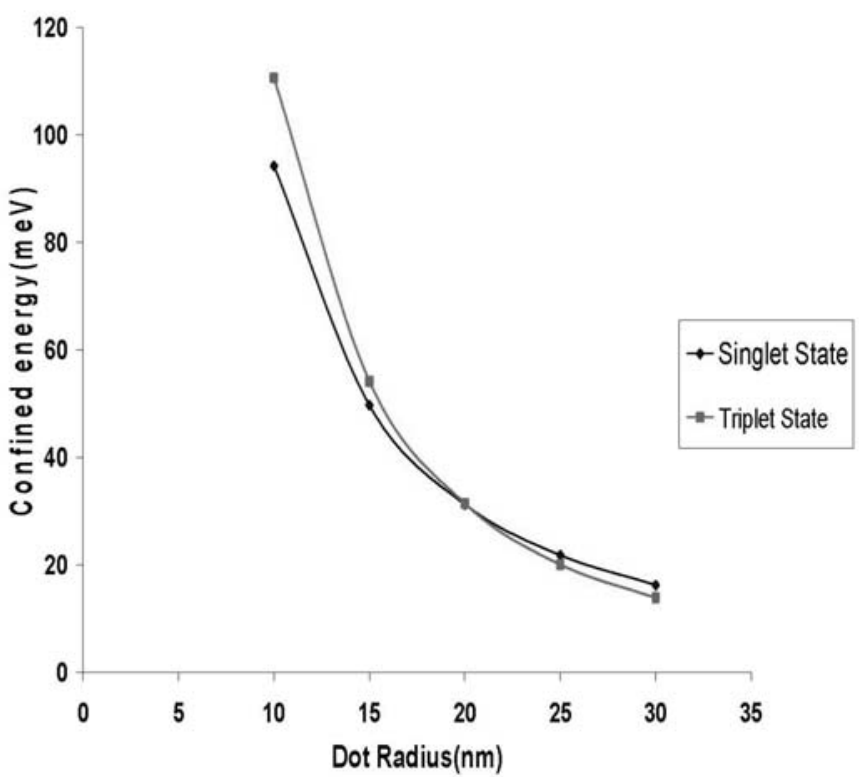

FIG. 2: Confined energy for the two electron system in a QD.

It is found that the singlet state confined energy is lower than that of the triplet for narrow dots which agrees with the quantum Monte Carlo EMA(Effective Mass Approximation) calculation as in Ref. [16]. The results show that the correlation effects are important for smaller dots and should be considered in the studies on all the low dimensional semi conducting systems.

The crossing of the singlet and triplet state energies at $R \simeq$ $20 \mathrm{~nm}$, from Fig. 2 is consistent with the Hartree-Fock calculation of Szafran et al. [7]. It is termed as "fictitious crossing" which is attributed to numerical accuracies [7]. At the point of crossing, $2 E_{1 s}+\Delta E_{s c}=E_{1 s}+E_{1 p}+\Delta E_{t c}$, where $\Delta E_{s c}$ and $\Delta E_{t c}$ are correlation energies in the singlet and triplet states. It immediately follows that $E_{1 p}-E_{1 s}=\Delta E_{s c}-\Delta E_{t c}$. Noting that $\Delta E_{t c}$ is negative, $E_{1 p}>E_{1 s}$ as expected. However, as $R \rightarrow \infty, E_{1 p} \rightarrow E_{1 s}$ and $\Delta E_{s c} \rightarrow \Delta E_{t c}$. In fact, the correlation energies themselves approach zero as is evident from Fig. 2. Since the $1 \mathrm{~s}$ and $1 \mathrm{p}$ states energies are degenerate as $R \rightarrow \infty$, whether a singlet state is favoured or a triplet state? By Hund's rule, a triplet state is expected without violating the Pauli principle. We believe that a spin flip Raman scattering experiment may throw more light on the nature of the ground state for $R>20 \mathrm{~nm}$ dots $[17,18]$.

\section{CONCLUSIONS}

The important conclusions that emerge from the present work are the following. a) The triplet state correlation energy is negative which is to be expected since the anti-symmetric spatial part of the wave function corresponds to the exchange 
interaction among the electrons. b)Both the singlet and the triplet energies decreases in magnitude as the dot radius approaches infinity. c) For smaller dots of radii lesser than 20nm the singlet state is favoured as it corresponds to the lowest energy state for the two electron system. However, for dot radii greater than $20 \mathrm{~nm}$, the triplet state is favoured con- sistent with the Pauli's exclusion principle and Hund's rules. d) A similar behaviour in the triplet and singlet state energies was observed in Szafran et al. [7] which they attributed to inaccuracies in the calculations. However, as we have explained above this crossing does not seem to be fictitious but has a physical basis.
[1] D. Gammon, Nature 405, 899 (2000).

[2] S. Bednarek, B. Szafran, and J.Adamowski, Phys. Rev. B 59, 13036 (1999).

[3] S.M. Maurer, S.R. Patal, C.M. Marcus, Phys. Rev.Lett. 83, 1403 (1999)

[4] M.A. Kastner, Ann.Phys.(Leipzig) 09, 885 (2000).

[5] G. Parascandolo, et al. Phys. Rev. B, 68, 245318 (2003).

[6] L. He, G. Bester, A. Zunger, Phys. Rev. B, 72, 195307 (2005).

[7] B. Szafran, J. Adamowski, S. Bednarek, Physica E, 5, (2000).

[8] A. Alavi, J.Chem.Phys. 113, 7735 (2000).

[9] A. Sivakami and K. Navaneethakrishanan, Physica E, 40, 649 (2008).

[10] M. R. Spiegel, Advanced Calculus, (Schaum's series), McGraw-Hill (1985).

[11] O. Madelung Ed., Semiconductor basic data, (2nd Revised Edition),Springer-verlag p .103 (1996).
[12] Sr. Gerardin Jeyam and K. Navaneethakrishnan, Solid state Communications, 126, 681 (2003).

[13] V.V. Mitin, V.A. Kochelap, M.A. Stroscio, Micro Electronics and Optoelectronics, Cambridge University Press, Cambridge, 1999.

[14] Y. Li, O.Voskoboynikov, J-L.Liu, C.P.Lee and S.M.Sze Nanotech, 1, 562 (2001).

[15] S. Bednarek, T. Chwiej, J. Adamowski, and B. Szafran, Phys. Rev. B, 67, 205316( 2003).

[16] C. Yannouleas and U.Landman, J.Phys.:Condens. Matter, 14, 1591 (2002).

[17] M. Nawrocki, R. Pland, G. Fishman and R. Galazka, Phys.Rev.Lett., 46735 (1981).

[18] A.V. Khaetskii and Y.V. Nazanov, Phys.Rev., B 64125316( 2001). 